\title{
Using the Particle Filter as Mitigation to GPS Vulnerability for Navigation at Sea
}

\author{
Rickard Karlsson, Fredrik Gustafsson, \\ Division of Automatic Control \\ Department of Electrical Engineering \\ Linköpings universitet, SE-581 83 Linköping, Sweden \\ WWW: http://www. control.isy.liu.se \\ E-mail: rickard@isy.liu.se, fredrik@isy.liu.se,
}

20th September 2005

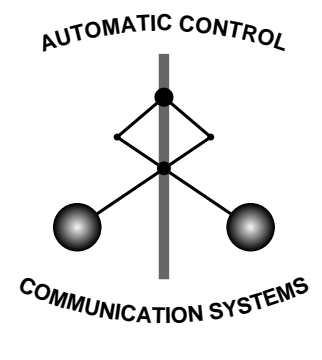

LINKÖPING

Report no.: LiTH-ISY-R-2699

Submitted to IEEE Statistical Signal Processing workshop 2005

Technical reports from the Control \& Communication group in Linköping are available at http://www. control.isy.liu.se/publications. 


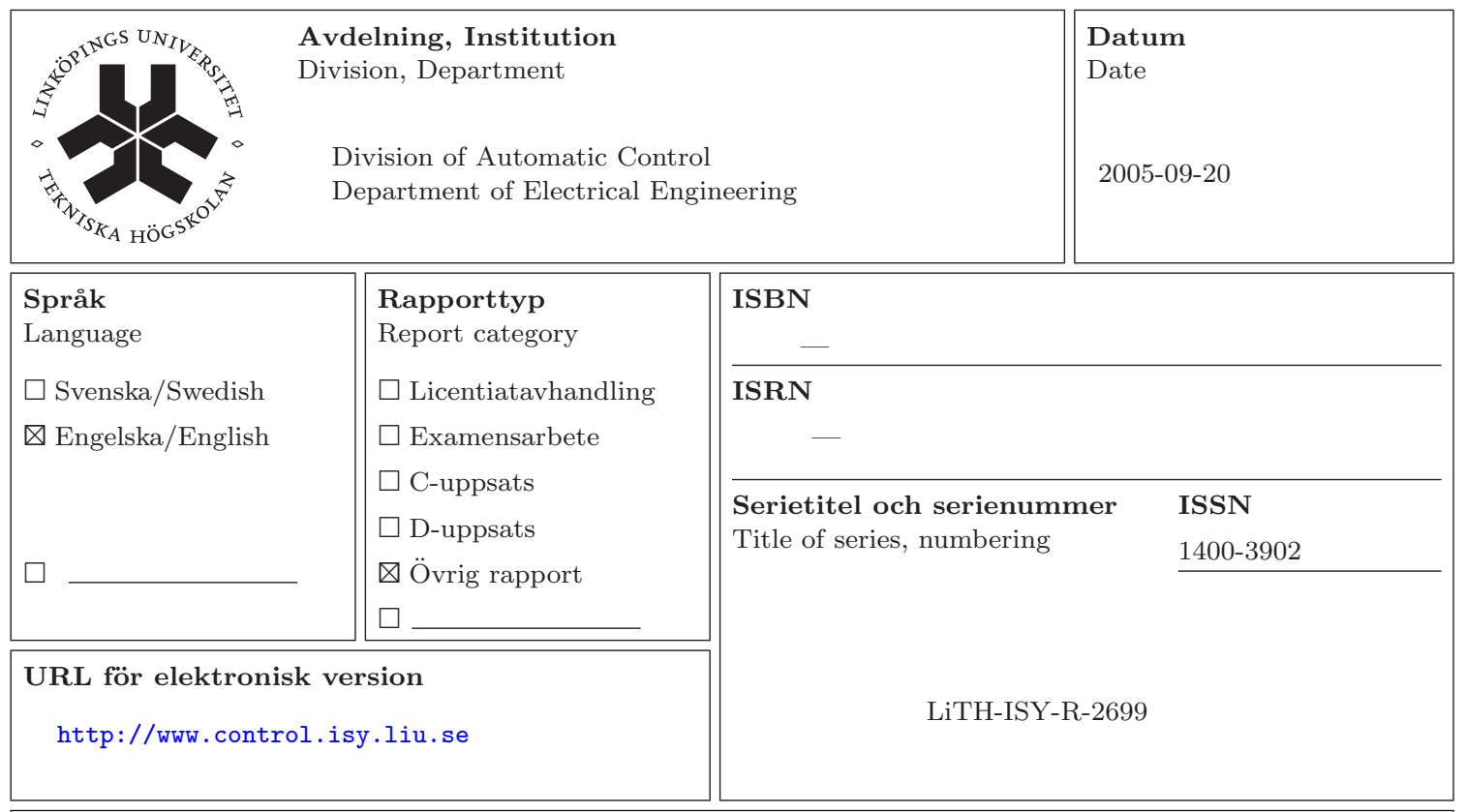

Titel Using the Particle Filter as Mitigation to GPS Vulnerability for Navigation at Sea

Title

Using the Particle Filter as Mitigation to GPS Vulneability for Navigation at Sea

Författare Rickard Karlsson, Fredrik Gustafsson,

Author

\section{Sammanfattning}

Abstract

A map-aided navigation method for positioning at sea is proposed, as a supplement to satellite navigation based on the global positioning system (GPS). The proposed Bayesian navigation method is based on information from a radar and information from databases. For the described system, the fundamental navigation performance expressed as the Cramér-Rao lower bound (CRLB) is analyzed and an analytic solution as a function of the position is derived. As a solution to the recursive Bayesian navigation problem, the particle filter is proposed. In an extensive Monte Carlo simulation performance equals a GPS system. 


\title{
Using the Particle Filter as Mitigation to GPS Vulnerability for Navigation at Sea
}

\author{
Rickard Karlsson and Fredrik Gustafsson \\ Department of Electrical Engineering \\ Linköping University,Sweden \\ E-mail: $\{$ rickard, fredrik\}@isy.liu.se
}

\begin{abstract}
A map-aided navigation method for positioning at sea is proposed, as a supplement to satellite navigation based on the global positioning system (GPS). The proposed Bayesian navigation method is based on information from a radar and information from databases. For the described system, the fundamental navigation performance expressed as the CramérRao lower bound (CRLB) is analyzed and an analytic solution as a function of the position is derived. As a solution to the recursive Bayesian navigation problem, the particle filter is proposed. In an extensive Monte Carlo simulation performance equals a GPS system.
\end{abstract}

Index Terms-Sea navigation, Particle filter, Cramér-Rao lower bound.

\section{INTRODUCTION}

$\mathbf{M}$ ODERN sea navigation systems are often based on satellite information from the global positioning system (GPS). For critical navigation applications, this sensor cannot be the only positioning sensor. In military applications, an independent backup sensor insensitive to GPS jamming is preferable. Even for civil applications the robustness against jamming may constitute one of the main design issues in the future. In [1], [2] the problem of intentional or unintentional GPS jamming is discussed and alternative backup systems are strongly recommended. This is discussed further in [3] where both bathymetric and celestial methods are described as alternatives to GPS navigation. At sea, the satellite signal is often received without problem, since there is a free lineof-sight to several satellites. However, under severe weather conditions, such as ice-building on the antenna, or due to the landscape geometry, or a system failure, GPS signals may not be available.

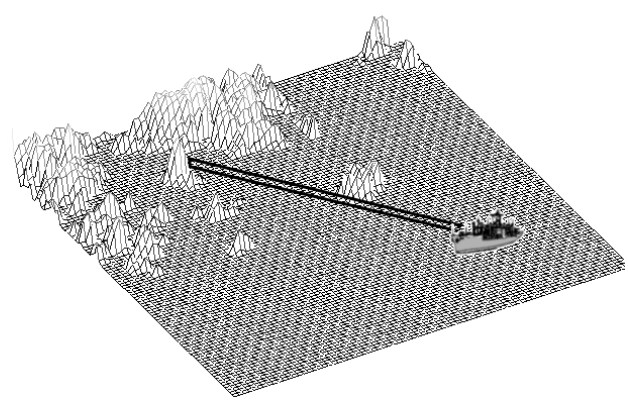

Fig. 1. The surface navigation with a radar sensor and a sea chart database.
As an alternative to satellite navigation it is possible to use terrain information together with sensor information from a distance measuring equipment (DME), such as a conventional radar. By comparing the terrain information from a database with the received DME measurements it is in many cases possible to get an accurate position estimate.

\section{NAVIGATION MODELS}

In this section the model used for surface navigation is presented. The system dynamics and the measurement relation are discussed.

\section{A. Motion Model}

Depending on the configuration, different sensors can be used, such as speedometers and accelerometers. Hence, the motion can be modeled using as many position derivatives as desired. Here, only longitudinal and lateral motion is considered, where the speed is measured. Consider the following state variables: Cartesian position $(X, Y)$, and crab angle, $\delta$, that is the angle between the velocity vector and the stem of the ship,

$$
x_{t}=\left(\begin{array}{lll}
X_{t} & Y_{t} & \delta_{t}
\end{array}\right)^{T}
$$

as depicted in Fig. 2. The following discrete time model with sample time $T$ is used for the navigation system

$$
x_{t+1}=f\left(x_{t}, u_{t}, w_{t}\right)=\left(\begin{array}{c}
X_{t}+v_{t} T \sin \left(\varphi_{t}-\delta_{t}\right) \\
Y_{t}+v_{t} T \cos \left(\varphi_{t}-\delta_{t}\right) \\
\delta_{t}
\end{array}\right)+w_{t},
$$

with input signal $u_{t}=\left(\begin{array}{llll}v_{t} & \varphi_{t} & \theta_{t} & \phi_{t}\end{array}\right)^{T}$, consisting of speed, $v_{t}$, compass, $\varphi_{t}$, elevation angle, $\theta_{t}$ and azimuth angle, $\phi_{t}$ relative to the ship's stem. The sensor azimuth angle, $\phi_{t}$, and elevation angle, $\theta_{t}$, are not present in the dynamic model, but will be used in the measurement relation described in Section II-B. The process noise, $w_{t}$, is considered independent and describes the model uncertainty. Note that here, the known input signal is in practice values obtained from sensors. Hence, if they are considered as noisy measurements, the process noise will also describe the uncertainty in the input. If $\delta$ is negligible or known the model simplifies even further to only position states. More advanced dynamic models can also be used, for instance a coordinated turn model, which was the case in [4]. For an overview of possible motion models, see the survey in [5]. 


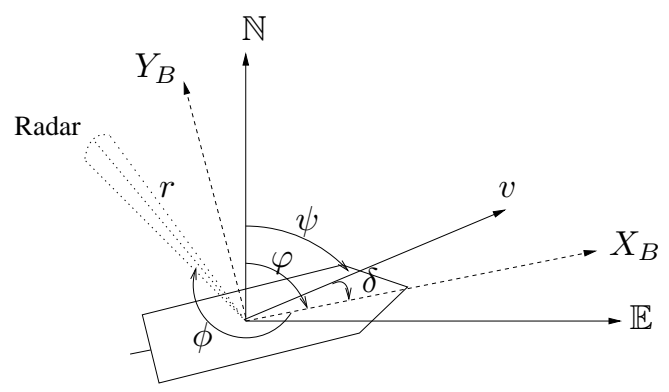

Fig. 2. The ship with compass angle $\varphi$ defined from the north direction $(\mathbb{N})$, together with the ship's body coordinate frame $\left(X_{B}, Y_{B}\right)$, crab angle $\delta$, velocity $v$ and heading $\psi$. The radar lobe is given with azimuth angle $\phi$ and the range $r=r\left(X, Y, \phi^{N}\right)$, where $\phi^{N}=\varphi+\phi$.

\section{B. Measurement Model}

For the surface navigation the range to land objects is measured by a radar sensor, (DME). Hence, the measurement relation is given by

$$
y_{t}=h\left(x_{t}, u_{t}\right)+e_{t}=r\left(x_{t}, \phi_{t}^{N}, \theta_{t}\right)+e_{t},
$$

where $r\left(x_{t}, \phi_{t}^{N}, \theta_{t}\right)$ is the measured range from position $x_{t}$ and with the sensor azimuth angle, $\phi_{t}^{N}=\varphi_{t}+\phi_{t}$, relative to north. The radar angle is defined in Fig. 2.

\section{Navigation System}

In Fig. 3 the complete surface or underwater navigation system, together with way-point calculation and auto pilot, is depicted. Depending on the application, one or more of the presented sensors are available. For surface navigation, the GPS signal can in many cases be the prime navigation sensor, where the estimate from the map-aided navigation method can be used to monitor performance. The main objective in this paper is to analyze the map-aided navigation method, hence the GPS part is not further discussed.

\section{THE CRAMÉR-RAO LOWER BOUND (CRLB)}

The main objective in this section is to find fundamental limits for the estimation performance expressed in terms of the system properties, for instance as a function of the measurement noise or the information available in the sea chart. The Cramér-Rao lower bound (CRLB) is such a characteristic for the second order moment [6]. This is done considering a static and dynamic case respectively. In the first case, only the measurement model is considered. In the second, the complete system dynamics is analyzed. The idea in this section is to perform a local analysis in each point in the sea chart and use that to analyze the global behavior.

In the sequel, the CRLB analysis is heavily based on expressions involving gradients of scalar functions or vector valued functions:

$$
\nabla_{x} g^{T}(x)=\left(\begin{array}{ccc}
\frac{\partial g_{1}}{\partial x_{1}} & \ldots & \frac{\partial g_{m}}{\partial x_{1}} \\
\vdots & & \vdots \\
\frac{\partial g_{1}}{\partial x_{n}} & \cdots & \frac{\partial g_{m}}{\partial x_{n}}
\end{array}\right), g: \mathbb{R}^{n} \mapsto \mathbb{R}^{m} .
$$

Also, the Laplacian for $g(x, y)$ with $x \in \mathbb{R}^{n}, y \in \mathbb{R}^{m}$ is defined as

$$
\Delta_{y}^{x} g(x, y)=\nabla_{y}\left(\nabla_{x} g(x, y)\right)^{T}, g: \mathbb{R}^{n} \times \mathbb{R}^{m} \mapsto \mathbb{R} .
$$

The theoretical posterior CRLB for a general dynamic system was derived in [7], [8], [9], [10]. The general state space model can be used to derive fundamental limits for navigation performance. In many applications, $\delta_{t}$ is small and can be discarded from the analysis, or it is known. Hence, for the CRLB analysis the following simplified model is used

$$
\begin{aligned}
x_{t+1} & =x_{t}+u_{t}+w_{t}, \\
y_{t} & =h\left(x_{t}, u_{t}\right)=r\left(x_{t}, \phi_{t}^{N}\right)+e_{t},
\end{aligned}
$$

where the horizontal position state vector, $x_{t} \in \mathbb{R}^{2}$, and the input signal, $u_{t}$, are defined as

$$
x_{t}=\left(\begin{array}{c}
X_{t} \\
Y_{t}
\end{array}\right), u_{t}=\left(\begin{array}{c}
v_{t} \sin \left(\varphi_{t}\right) \\
v_{t} \cos \left(\varphi_{t}\right)
\end{array}\right),
$$

assuming independent additive process noise $w_{t}$. The observation relation consists of range measurements with measurement noise $e_{t}$. Using standard notations, consider independent noise sources, with variances $Q_{t}=\operatorname{Cov}\left(w_{t}\right)$ and $R_{t}=$ $\operatorname{Cov}\left(e_{t}\right)$, the posterior CRLB for filtering can be written as, [9]

$$
P_{t+1 \mid t+1}^{-1}=Q_{t}^{-1}+J_{t+1}-S_{t}^{T}\left(P_{t \mid t}^{-1}+V_{t}\right)^{-1} S_{t},
$$

where

$V_{t}=\mathbb{E}\left(-\Delta_{x_{t}}^{x_{t}} \log p\left(x_{t+1} \mid x_{t}\right)\right), Q_{t}^{-1}=\mathbb{E}\left(-\Delta_{x_{t+1}}^{x_{t+1}} \log p\left(x_{t+1} \mid x_{t}\right)\right)$, $S_{t}=\mathbb{E}\left(-\Delta_{x_{t}}^{x_{t+1}} \log p\left(x_{t+1} \mid x_{t}\right)\right), J_{t}=\mathbb{E}\left(-\Delta_{x_{t}}^{x_{t}} \log p\left(y_{t} \mid x_{t}\right)\right)$,

where the bound is given by

$$
\operatorname{Cov}\left(x_{t}-\hat{x}_{t \mid t}\right)=\mathbb{E}\left(\left(x_{t}-\hat{x}_{t \mid t}\right)\left(x_{t}-\hat{x}_{t \mid t}\right)^{T}\right) \succeq P_{t \mid t} .
$$

If the model in (6) is considered with Gaussian process noise, it gives $S_{t}=V_{t}=Q_{t}^{-1}$. Hence, given the true trajectory for the state vector the posterior bound can be calculated.

In order to analyze the performance, without performing any simulations, a local analysis of the dynamic system is conducted. The assumption is that the covariance should reach a stationary value, i.e., consider a point $x^{0}$ and assume $\bar{P}(x)=$ $P_{t+1 \mid t+1}(x)=P_{t \mid t}(x)$, for all $x$ such that $\left|x-x^{0}\right|<\epsilon$. Using this assumption in (8), it is possible to obtain an expression for $\bar{P}(x)$. This is done by applying the matrix inversion lemma

$$
(A+B C D)^{-1}=A^{-1}-A^{-1} B\left(D A^{-1} B+C^{-1}\right)^{-1} D A^{-1},
$$

using $A=Q, B=D=\mathcal{I}$ and $C=\bar{P}(x)$, where $\mathcal{I}$ is the identity matrix. This gives

$$
\bar{P}^{-1}(x)=(\bar{P}(x)+Q)^{-1}+J(x),
$$

Solving for $J(x)$ and applying the matrix inversion lemma again with $A=B=D=\bar{P}(x)$ and $C=Q^{-1}$ yield

$$
\begin{aligned}
& J(x)=\bar{P}^{-1}(x)-(\bar{P}(x)+Q)^{-1}= \\
& \left(\bar{P}(x)+\bar{P}(x) Q^{-1} \bar{P}(x)\right)^{-1} .
\end{aligned}
$$




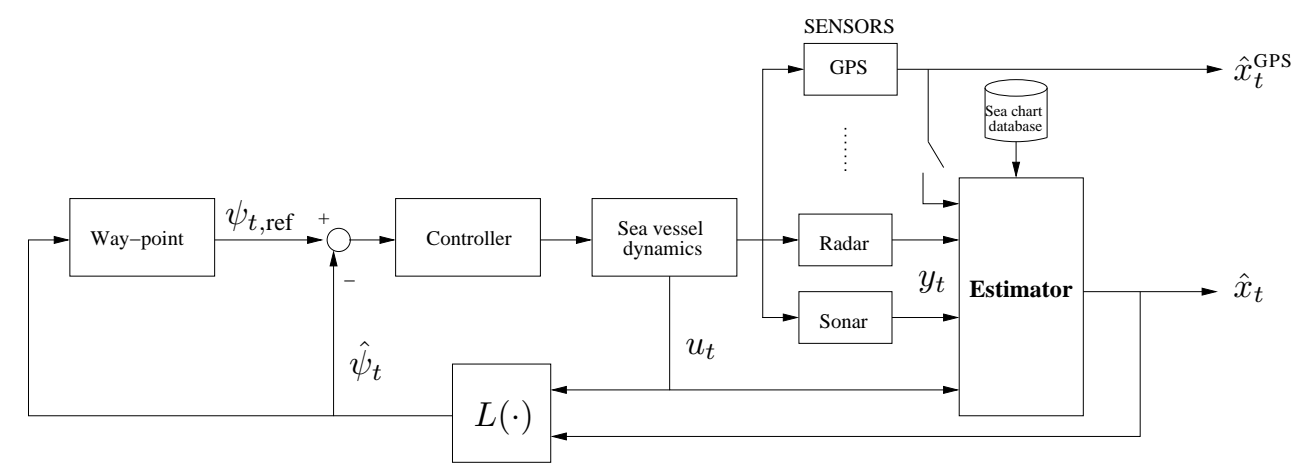

Fig. 3. The complete navigation system, used in the applications in Section V, consisting of a way-point unit, a controller, the sea chart database, the estimator and various sensors. The reference is given by the desired heading, $\psi_{t, \text { ref }}$ and the estimated heading is given as $\hat{\psi}_{t}=L\left(\hat{x}_{t}, u_{t}\right)$.

Hence, if $J$ is assumed invertible, then

$$
\bar{P}(x)+\bar{P}(x) Q^{-1} \bar{P}(x)-J(x)^{-1}=0 .
$$

Completing squares gives

$$
\begin{array}{r}
\left(\bar{P}(x) Q^{-1 / 2}+\frac{1}{2} Q^{1 / 2}\right)\left(Q^{-1 / 2} \bar{P}(x)+\frac{1}{2} Q^{1 / 2}\right) \\
=J(x)^{-1}+\frac{1}{4} Q .
\end{array}
$$

Multiply with $Q^{-1 / 2}$ from left and right in the expression

$$
\begin{aligned}
\left(Q^{-1 / 2} \bar{P}(x) Q^{-1 / 2}+\right. & \left.\frac{1}{2} \mathcal{I}\right)\left(Q^{-1 / 2} \bar{P}(x) Q^{-1 / 2}+\frac{1}{2} \mathcal{I}\right) \\
& =Q^{-1 / 2} J(x)^{-1} Q^{-1 / 2}+\frac{1}{4} \mathcal{I} .
\end{aligned}
$$

Since all matrices are symmetric, a unique matrix square root can be defined. Hence, solving for the symmetric and positive definite matrix, $\bar{P}(x)>0$, now yields

$Q^{-1 / 2} \bar{P}(x) Q^{-1 / 2}+\frac{1}{2} \mathcal{I}=\left(Q^{-1 / 2} J(x)^{-1} Q^{-1 / 2}+\frac{1}{4} \mathcal{I}\right)^{1 / 2}$

Hence, the explicit solution to the Ricatti equation (11) is

$\bar{P}(x)=-\frac{1}{2} Q+Q^{1 / 2}\left(Q^{-1 / 2} J(x)^{-1} Q^{-1 / 2}+\frac{1}{4} \mathcal{I}\right)^{1 / 2} Q^{1 / 2}$,

where $Q$ and $J(x)$ are defined below (8).

\section{RECURSIVE BAYESIAN ESTIMATION}

Navigation problems are often treated as Bayesian inference. The two map aided navigation methods described in Section II are described by nonlinear problems. Consider the following general state-space model

$$
\begin{aligned}
x_{t+1} & =f\left(x_{t}, u_{t}, w_{t}\right), \\
y_{t} & =h\left(x_{t}\right)+e_{t},
\end{aligned}
$$

where $x_{t} \in \mathbb{R}^{n}$ denotes the state of the system, $u_{t}$ the input signal and $y_{t}$ the observation at time $t$. The process noise $w_{t}$ and measurement noise $e_{t}$ are assumed independent with densities $p_{w_{t}}$ and $p_{e_{t}}$ respectively. Let $\mathbb{Y}_{t}=\left\{y_{i}\right\}_{i=0}^{t}$ be the set of observations until present time.
The Bayesian estimation problem is given by, [11],

$$
\begin{aligned}
p\left(x_{t+1} \mid \mathbb{Y}_{t}\right) & =\int_{\mathbb{R}^{n}} p\left(x_{t+1} \mid x_{t}\right) p\left(x_{t} \mid \mathbb{Y}_{t}\right) d x_{t}, \\
p\left(x_{t} \mid \mathbb{Y}_{t}\right) & =\frac{p\left(y_{t} \mid x_{t}\right) p\left(x_{t} \mid \mathbb{Y}_{t-1}\right)}{p\left(y_{t} \mid \mathbb{Y}_{t-1}\right)},
\end{aligned}
$$

where $p\left(x_{t+1} \mid \mathbb{Y}_{t}\right)$ is the prediction density and $p\left(x_{t} \mid \mathbb{Y}_{t}\right)$ the filtering density. The problem is in general not analytically solvable. To solve the non-tractable Bayesian estimation problem in an on-line application without using linearization or Gaussian assumptions, the particle filter, [10], [12], can be used. In Section $\mathrm{V}$ the sequential importance sampling (SIS) version of the particle filter is applied.

\section{Applications}

The navigation system is presented in Fig. 3, where the ship is equipped with a radar, measuring relative distance to any land object. The sensor is assumed stabilized or that the deviation angle is small relative to the uncertainty in the radar sensor. To simplify the analysis, the speed relative to ground and the compass angle are considered as input signals, i.e., noiseless measurements, as described in Section II-A. However, this assumption is not necessary, and by introducing them as states-variables in the model, these can be estimated. Crucial for the positioning algorithm is a comparison of relative range measurements from the radar with expected land areas from a sea chart database. This is done in a statistically optimal way, using the particle filter. The map presented in Fig. 4 is computed from a vectorized sea chart.

Since the speed is rather small compared to the radar revolution, a complete radar picture can be processed for each time the filter is updated. In order to reduce the amount of possible range data, only a fixed number, $(M)$, of radar strobes are considered each revolution. The radar produces many range measurements in any given direction. In the algorithm, only the measurement closest to the ship is considered. The database consists of a sea chart. In Fig. 4 the scenario is presented, where the ship's true position and some radar measurements are depicted. Also, the probability density function for each coordinate is given. As seen, after only 5 revolutions of the radar an accurate position is given. The initial distribution for the example given in Fig. 4 was uniformly distributed around the true position, $\pm 800 \mathrm{~m}$ in each direction. 

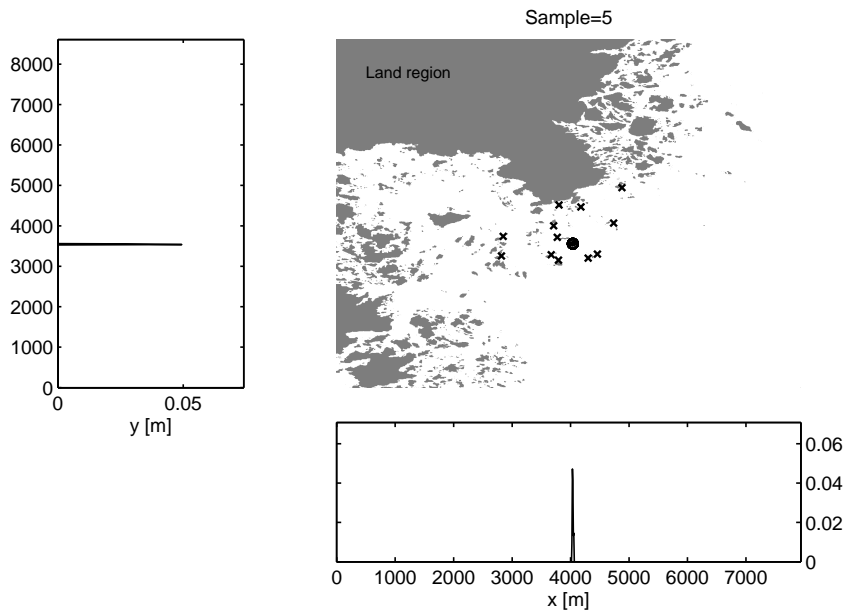

Fig. 4. The true position (o) and radar measurements (x), together with the particle cloud. The marginalized pdf for the $X$ and $Y$ directions are also shown.

In a Monte Carlo simulation study the scenario given previously is used. A straight own-ship trajectory is used in all simulations with different measurement noise realizations. However, theoretical studies based on (17), indicates that any trajectory in the sea chart gives sufficient positioning performance. In Fig. 5 the RMSE as a function of time is given for 50 Monte Carlo simulations. The parameters used in the Monte Carlo evaluation are presented in Table I. The filter is initialized with a large amount of particles, but decreased after a short time in order to reach real-time performance on a desktop computer. This simplifies the initialization of the algorithm. The number of particles can be reduced even further, but in order to avoid divergence in any of the Monte Carlo simulations the final number is chosen to $N=10000$. As seen in Fig. 5 the RMSE from the Monte Carlo simulations

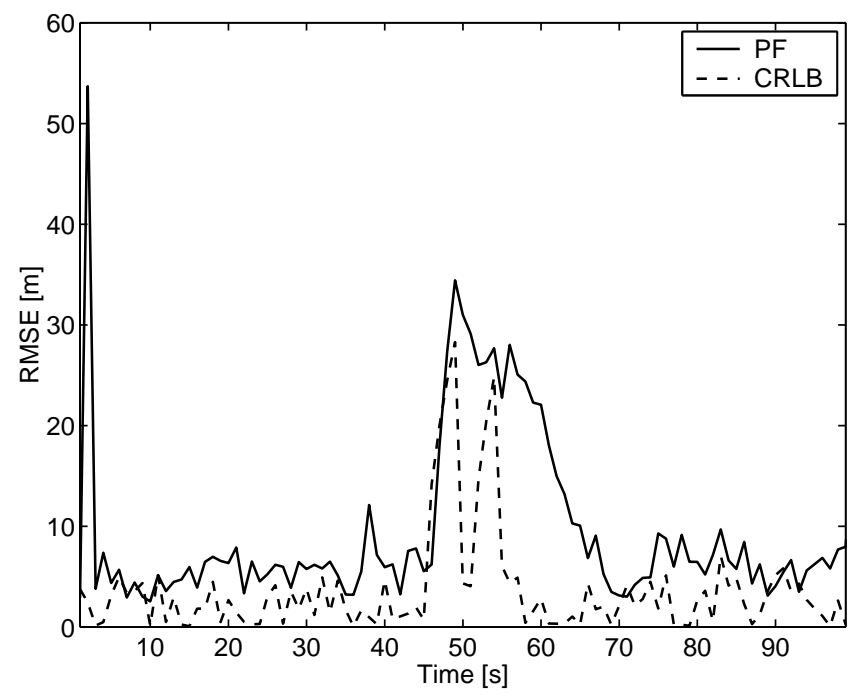

Fig. 5. Surface navigation position RMSE(t) for the particle from 50 Monte Carlo simulations together with the CRLB limit as the solution of the EKF around the true trajectory.

are close to the fundamental limit. The performance using the map-aided navigation method equals or is slightly better than an ordinary GPS based navigation system, when there are sufficient returns from land objects. Also note that the peak is due to a region when very few of the radar strobes (from the downsampled radar picture) actually reflect any land area.

TABLE I

SURFACE NAVIGATION PARAMETERS

\begin{tabular}{ll}
\hline \hline Monte Carlo simulations & 50 \\
Process noise covariance & $Q=\operatorname{diag}\left(10^{2}, 10^{2}, 0^{2}\right)$ \\
Measurement noise covariance & $R=10^{2}$ \\
Max radar meas./revolution & $M=16$ \\
Sample time & $T=1$ \\
Number of particles & $N=50000 \searrow N=10000$ \\
Radar interval & $R_{\min }=300, R_{\max }=6000$ \\
\hline \hline
\end{tabular}

\section{CONCLUSIONS}

In this paper a framework for sea navigation using sea chart information and a distance measuring equipment is developed. The navigation performance is analyzed both theoretically and using Monte Carlo simulations. An analytic Cramér-Rao lower bound for the proposed navigation model has been derived. In an extensive Monte Carlo simulation the system reaches GPS performance using only a radar sensor and the map-aided navigation method.

\section{ACKNOWLEDGMENT}

The authors would like to thank: the Swedish Maritime Administration (Sjöfartsverket) for providing vector based sea chart information. Bengt Ståhl for initial encouragement and for pointing out the references, [1], [2]. Erik Svensson for assisting with sea chart vector data transformations.

\section{REFERENCES}

[1] J. Volpe, "Vulnerability assessment of the transportation infrastructure relying on global positioning system final report," U.S. Department of Transportation. National Transportation Systems Center, Tech. Rep., Aug. 2001. [Online]. Available: www.navcen.uscg.gov/archive/2001/ Oct/FinalReport-v4.6.pdf

[2] "GNSS vulnerability \& mitigation measures. A study for the European maritime radionavigation forum (rev. 6)," Tech. Rep., 2001.

[3] F. Pappalardi, S. J. Dunham, M. E. LeBlang, T. E. Jones, J. Bangert, and G. Kaplan, "Alternatives to GPS," in Proceedings of the MTS/IEEE Conference and Exhibition (OCEANS'01), vol. 3, Nov. 2001, pp. 14521459.

[4] R. Karlsson and F. Gustafsson, "Particle filter for underwater terrain navigation," in IEEE Statistical Signal Processing Workshop, St. Louis, MO, USA, Oct. 2003, pp. 526-529, invited paper.

[5] X. R. Li and V. Jilkov, "A survey of maneuvering target tracking: Dynamics models," in Proceedings of SPIE Conference on signal and data processing of small targets, vol. 4048, Apr. 2000, pp. 212-235.

[6] S. Kay, Fundamentals of Statistical Signal Processing. Prentice Hall, 1993.

[7] H. L. Van Trees, Detection, Estimation and Modulation Theory. New York: Wiley, 1968.

[8] P. Tichavsky, P. Muravchik, and A. Nehorai, "Posterior Cramér-Rao bounds for discrete-time nonlinear filtering," IEEE Trans. Signal Processing, vol. 46, no. 5, pp. 1386-1396, 1998.

[9] N. Bergman, "Recursive Bayesian estimation: Navigation and tracking applications," Linköping Studies in Science and Technology. Dissertations No. 579, Linköping University, Linköping, Sweden, 1999.

[10] A. Doucet, N. de Freitas, and N. Gordon, Eds., Sequential Monte Carlo Methods in Practice. Springer Verlag, 2001.

[11] A. H. Jazwinski, Stochastic Processes and Filtering Theory, ser. Mathematics in Science and Engineering. Academic Press, 1970, vol. 64.

[12] N. J. Gordon, D. J. Salmond, and A. Smith, "A novel approach to nonlinear/non-Gaussian Bayesian state estimation," in IEE Proceedings on Radar and Signal Processing, vol. 140, 1993, pp. 107-113. 